\title{
RELACIÓN ENTRE TRAUMA, DISOCIACIÓN Y SÍNTOMAS PSICótTiCos POSITIVOS
}

\section{RELATIONSHIP OF TRAUMA, DISSOCIATION, AND POSITIVE PSYCHOTIC SYMPTOMS}

\author{
María del Pilar Castro-FernándeZ ${ }^{1}$, SAlVAdOR Perona-GARCelán ${ }^{1,2}$, \\ CRISTINA SENÍN-CALDERÓN ${ }^{3}$ Y JUAN F. RODRÍGUEZ-TESTAL ${ }^{1}$
}

Cómo referenciar este artículo/How to reference this article:

Castro-Fernández, M. P., Perona-Garcelán, S., Senín-Calderón, C. y Rodríguez-Testal, J. F. (2015). relación entre trauma, disociación y síntomas psicóticos positivos [Relationship of Trauma, Dissociation, and Positive Psychotic Symptoms]. Acción Psicológica, 12(2), 95-108. doi: http://dx.doi.org/10.5944/ap.12.2.15824

\section{Resumen}

El objetivo de esta investigación fue estudiar experiencias traumáticas y disociativas en una muestra de pacientes españoles con psicosis y su relación con las alucinaciones. Setenta y un pacientes con psicosis completaron una escala de experiencias disociativas (DES-II, Carlson y Putnam, 1993), un cuestionario de traumas (TQ, Davidson, Hughes y Blazer, 1990) y se les administró también los ítems de delirios y alucinaciones de la PANSS (Kay, Opler y Lindenmayer, 1988). Los resultados mostraron que los sujetos que presentaban alucinaciones y delirios habían experimentado un número significativamente mayor de las experiencias traumáticas en la infancia, pero no en la edad adulta. Con respecto a la disociación, los sujetos con alucinaciones y delirios presentaban puntuaciones altas en disociación que los sujetos sin estos síntomas

Correspondencia: Salvador Perona-Garcelán. Departamento de Personalidad, Evaluación y Tratamiento Psicológicos, España, Universidad de Sevilla (US), España.

Email: sperona@us.es

${ }^{1}$ Universidad de Sevilla (US), España.

${ }^{2}$ Hospital Universitario Virgen del Rocío, España.

${ }^{3}$ Universidad de Cádiz (UCA), España.

Recibido: 11 de septiembre de 2015

Aceptado: 30 de octubre de 2015 
psicóticos. Respecto al tipo de trauma, se encontró que los sujetos con alucinaciones presentaban más abusos físicos y amenazas en la infancia, no encontrándose diferencias en el tipo de traumas en función de la presencia o no de delirios. Por último, de los factores estudiados en esta investigación, solamente la despersonalización predijo la presencia de alucinaciones, encontrándose que ninguno de dichos factores predijo la presencia de delirios.

Palabras clave: alucinación; delirios; trauma; disociación; despersonalización.

\begin{abstract}
The purpose of this research was to study traumatic and dissociative experiences in a sample of Spanish patients with psychosis and their relationship to hallucinations. Seventy-one patients with psychosis filled in a dissociative experiences scale (DES-II, Carlson \& Putnam, 1993), a trauma questionnaire (TQ, Davidson, Hughes, \& Blazer, 1990) and the PANSS delusion and hallucination items (Kay, Opler, \& Lindenmayer, 1988). The results showed that subjects who had hallucinations and delusions had undergone a significantly larger number of traumatic experiences in childhood, but not as adults. Subjects with hallucinations and delusions had higher scores in dissociation than those who did not have these psychotic symptoms. It was also found that subjects with hallucinations had had experienced more physical abuse and threats in childhood, while there were no differences in the type of trauma for presence or not of delusions. Finally, of the factors studied in this research, only depersonalization predicted the presence of hallucinations, and none of these factors predicted the presence of delusions.
\end{abstract}

Keywords: hallucinations; delusions; trauma; dissociation; depersonalization.

\section{Introducción}

Actualmente hay un consenso entre investigadores que las alucinaciones auditivas no son estrictamente una experiencia perceptiva (percepciones sin objeto), sino que tienen que ver con procesos cognitivos en los que la persona no reconoce sus propios eventos internos (pensamientos, recuerdos, imágenes, etc.) o los atribuye a una fuente externa. Este tipo de experiencias se pueden presentar en personas con un diagnóstico de esquizofrenia o del espectro esquizofrénico, pero también en otros diagnósticos como en el trastorno afectivo bipolar y en depresiones unipolares, en trastornos por estrés postraumáticos, en trastornos disociativos y también en personas sin un trastorno psiquiátrico (ver Alemán y Larǿi, 2008).

Tradicionalmente hay dos grandes líneas de investigación; una de ellas parte del supuesto de que las alucinaciones se deben a un problema en el reconocimiento de los propios eventos internos provocado por un déficit cognitivo, en los que los mecanismos implicados serían los procesos neuropsicológicos que tienen que ver con la automonitorización de las propias acciones y pensamientos (e.g., Frith, 1995). La otra línea de investigación parte del supuesto de que se produce un sesgo cognitivo en la monitorización de los eventos internos, es decir, determinadas creencias metacognitivas dificultan los procesos de discriminación de la realidad (e.g., Bentall, 1990).

Recientemente, basándose en estas líneas de investigación, ha surgido un gran interés por estudiar los efectos de los traumas y las experiencias disociativas en personas con psicosis en general, y con las alucinaciones $\mathrm{y}$ delirios en particular.

Autores como Read (1997), proponen que la sintomatología psicótica puede emerger como reacción a un trauma, en el que la persona afectada experimenta determinados eventos vitales como peligrosos para su vida, o para su integridad física o psicológica. En personas con psicosis en general, Bendall, Jackson, Hulbert y McGorry (2008), en una revisión de 46 estudios realizados sobre este tema, encontraron que la prevalencia total de traumas en la infancia en grupos de personas con psi- 
cosis estaba en un rango entre $28 \%$ y $73 \%$; la de abusos sexuales en la infancia estaba entre un $13 \%$ y $61 \%$; y los abusos físicos estaban en un rango entre $10 \%$ y $61 \%$. En la edad adulta, la mayoría de los pacientes psiquiátricos sufren experiencia traumáticas del tipo de agresiones físicas graves. Un estudio encontró que el año previo a la hospitalización psiquiátrica, el $63 \%$ sufrieron violencia física de sus parejas y el $46 \%$ de los que vivían en sus hogares fueron agredidos por miembros de su familia (Cascardi, Mueser, Degiralomo y Murrin, 1996). Las agresiones también pueden ocurrir fuera del entorno familiar, incluyendo también a los profesionales de salud mental (Ritsher, Coursey y Farrell, 1997). La mayoría de las mujeres con psicosis han sufrido agresiones sexuales también en la edad adulta. Aproximadamente un tercio han sido violadas, y sobre una cuarta parte de los hombres han sido agredidos sexualmente siendo adultos (Read, Goodman, Morrison, Ross y Aderhold, 2004).

En cuanto a la relación específica con las alucinaciones, se ha encontrado en diversos estudios en personas con psicosis una correlación positiva con los traumas en la infancia (e.g., Perona-Garcelán et al., 2010; Read, van Os, Morrison y Ross, 2005), con la disociación (e.g., Kilcommons y Morrison, 2005; PeronaGarcelán et al., 2008), y más concretamente con la absorción y despersonalización (Perona-Garcelán, GarcíaMontes et al., 2012).

Sin embargo, existen pocos trabajos que hayan estudiado la relación entre trauma en la infancia y disociación en personas con psicosis. En todos ellos se destaca que los pacientes diagnosticados de psicosis, que han sufrido experiencias traumáticas en la infancia, puntúan más alto en una escala de experiencias disociativas que aquellos que no habían sufrido dicho tipo de trauma (p.ej., Perona-Garcelán et al., 2010; Ross y Keyes, 2004). Por otro lado, en algunos estudios se ha encontrado que en pacientes con esquizofrenia, la disociación está positivamente correlacionada con el abuso emocional y físico (Schäfer et al., 2006) y con el abuso sexual (Offen, Waller y Thomas, 2003). Esta asociación fue especialmente robusta para los abusos sexuales sobre otros tipos de traumas. En otro trabajo, Sar et al. (2010) encontraron también una asociación entre traumas en la infancia y disociación, pero no entre traumas en la infancia y síntomas psicóticos positivos.

En cuanto a los delirios, hay pocos estudios que hayan investigado la relación específica entre esta variable y traumas en la infancia. En ellos se han encontrado resultados menos robustos y contradictorios, en los que por un lado, algunos estudios han encontrado una asociación entre estas variables (Perona-Garcelán, Carrascoso-López et al., 2012), y otros no las han encontrado (PeronaGarcelán et al., 2010; Read, Agar, Argyle y Aderhold, 2003; Schenkel et al., 2005).

En cuanto al estudio de la relación entre traumas en la edad adulta y alucinaciones y delirios también hay poco estudios. Read et al. (2003), relacionaron los efectos del abuso sexual y físico en adultos con síntomas psicóticos específicos. Ellos encontraron que los abusos sexuales (pero no los físicos) estaban significativamente relacionados con las alucinaciones, delirios y trastornos formales del pensamiento, sólo cuando eran analizados conjuntamente con los abusos en la infancia. PeronaGarcelán et al. (2010) tampoco encontraron una asociación entre los traumas en la adultez y los delirios y las alucinaciones. En cuanto a la relación entre trauma en la edad adulta y disociación, Perona-Garcelán et al. (2010) no encontraron ninguna asociación entre estas dos variables.

Por tanto, parece que los traumas en la infancia presentan una asociación con las alucinaciones auditivas, sin embargo, hemos encontrado resultados no consistentes en cuanto a su relación con los delirios. Por otro lado, en cuanto a la relación entre traumas en la edad adulta, disociación y síntomas psicóticos positivos, apenas tenemos datos para poder llegar a conclusiones claras y robustas. Por ello, nos planteamos como objetivos en este trabajo, el estudio de la relación entre traumas (en la infancia y la adultez) con la disociación y los síntomas psicóticos positivos (alucinaciones auditivas y delirios) en una muestra de pacientes psicóticos españoles. Las hipótesis del presente estudio fueron las siguientes:

1. Los sujetos con alucinaciones auditivas y delirios presentarán en general más experiencias traumáticas en la infancia que los sujetos sin este tipo de síntomas psicóticos positivos. 
2. No encontraremos diferencias significativas entre sujetos con y sin alucinaciones auditivas y delirios en relación a las experiencias traumáticas padecidas en la edad adulta.

3. Los sujetos con alucinaciones auditivas y delirios presentaran más experiencias traumáticas de tipo interpersonal (abuso sexual y físico) en la infancia que los sujetos sin estos síntomas psicóticos positivos.

4. Los sujetos con alucinaciones auditivas y delirios tendrán mayores niveles de disociación que los sujetos sin estos síntomas psicóticos positivos.

5. Los sujetos que han sufrido traumas en la infancia tendrán mayores niveles de disociación que los sujetos sin experiencias traumáticas.

6. La despersonalización (frente a otros tipos de experiencias disociativas como la amnesia disociativa y la absorción y los traumas) predecirá la presencia de alucinaciones.

\section{Método}

\section{Participantes}

La muestra fue de un total de 71 pacientes que habían sido diagnosticados de psicosis según criterios del Manual Diagnóstico y Estadístico de los Trastornos Mentales (4a Ed., APA, 1994). Ninguno de ellos estaba hospitalizado en el momento de la realización de este estudio, y asistían a las actividades del centro de día de la Unidad de Rehabilitación de Salud Mental del Hospital Universitario Virgen del Rocío de Sevilla (España). Todos tenían prescrito tratamiento farmacológico por sus psiquiatras referentes de sus Unidades de Salud Mental Comunitarias. Las edades oscilaban de 20 a 54 años: la edad media total fue de 39.08 años $(D T=8.98)$; la edad media en los hombres fue $38.63(D T=9.15)$, y en las mujeres fue de 40.53 $(D T=8.50)$. La tasa de sujetos según el sexo era 17:54 mujeres y hombres, respectivamente.

\section{Instrumentos}

The Trauma Questionnaire (TQ, Davidson, Hughes y Blazer, 1990)

Para medir el número y tipo de traumas en la infancia y la edad adulta, utilizamos el listado de experiencias traumáticas recogidos en esta escala, en la que el sujeto solo tenía que señalar cuáles de las experiencias recogidas en dicho listado había sufrido a lo largo de su vida, registrándose el número de tales experiencias y la edad en la que se produjeron. Se eliminaron del análisis de los datos los ítems de esta escala que valoraban el impacto y síntomas provocados por las experiencias traumáticas, por no estar dentro de los objetivos de la presente investigación. En este estudio hemos definido como trauma en la infancia aquellas experiencias que ocurrieron hasta los 15 años de edad, y experiencias traumáticas en la edad adulta, las que se produjeron a partir de los 16 años. Debido a que en el listado no recogía de forma específica el ítem de abuso sexual, para incluir este tipo de experiencias en el análisis de datos se sumaron los ítems de violación, incesto y en el apartado de respuesta libre de "Otra situación" aquellas respuestas que tuviesen que ver con experiencias de abuso sexual. Se usó la versión española de esta escala, en la que el Alfa de Cronbach es .99 para la escala completa, .97 para la subescala de frecuencia de acontecimientos traumáticos, y .98 para la subescala de severidad (Bobes et al., 2000).

The Dissociative Experience Scale (DES-II, segunda versión, Carlson y Putnam, 1993)

Esta escala consiste en un cuestionario autoinformado de 28 ítems, diseñado para medir disociación en población clínica y también en población sin patología psiquiátrica. La respuesta a los ítems se hace mediante una escala de porcentajes, en la que se mide la frecuencia en la que ocurre en la vida diaria cada uno de los síntomas descritos. El sujeto puntúa $0 \%$ si nunca le ha ocurrido lo que se describe en ella y $100 \%$ si le ocurre con mucha frecuencia. Concretamente se utilizó en el presente estudio la adaptación española realizada por Icaran, Colom y Orengo-García (1996). El Alfa de Cronbach de la versión española es de .91. 
Tabla 1

Número y porcentaje de sujetos con traumas, número total de traumas y media (DT) de traumas vividos en la infancia y la edad adulta

\begin{tabular}{lll}
\hline Variables & Infancia & Adultez \\
\hline Número de pacientes con traumas & $32(45.1 \%)$ & $57(80.3 \%)$ \\
Número total de traumas & 84 & 152 \\
$M(D T)$ & $1.18(1.76)$ & $2.14(1.84)$ \\
\hline
\end{tabular}

The Positive and Negative Syndrome Scale (PANSS, Kay, Opler y Lindenmayer, 1988)

Es una entrevista semiestructurada para medir la presencia y gravedad de síntomas psicóticos positivos y negativos. En este estudio sólo se utilizaron los ítems de esta entrevista para medir las alucinaciones y los delirios. Las respuestas se puntúan según una escala ordinal de siete puntos, en el que 1 punto indica la ausencia de patología y 7 indica gravedad máxima del síntoma valorado. En este estudio se utilizó la versión española realizada por Peralta y Cuesta (1994). El Alfa de Cronbach es .73 para la escala positiva, de .83 para la negativa, y de .87 para la de psicopatología general.

\section{Procedimiento}

Después de solicitar a los pacientes el consentimiento informado para tomar parte en esta investigación, y de explicarles los objetivos del presente estudio, se les administró los ítems de delirios y alucinaciones de la PANSS, la DES-II y el TQ. Dependiendo del paciente, la duración aproximada de aplicación de todas estas pruebas fue aproximadamente de 45 minutos.

\section{Análisis de datos}

Siempre que ha sido necesario, por el tipo de variables empleadas en este estudio, se comprobó previamente la hipótesis de normalidad (prueba de Kolmogorov-Smirnov). Para la comparación de grupos independientes, en los casos en los que se verificó dicha hipótesis, se utilizó la $t$ de Student, y en los casos en los que no se verificó dicha hipótesis se aplicó la prueba $U$ de Mann-Whitney. Para la predicción de las alucinaciones con respecto a las experiencias disociativas y traumáticas, se aplicó también una regresión logística binaria, atendiendo a los criterios de ajuste la prueba de Hosmer y Lemeshow, y el estadístico de Wald.

\section{Resultados}

En la Tabla 1 se muestra el número de sujetos con traumas en la infancia y en la edad adulta, así como el número total y la media de los traumas experimentados por los participantes en estos dos períodos de la vida. Asimismo, en la Tabla 2 se muestra el número de sujetos según tipos de traumas en la infancia.

Tabla 2

Número de sujetos (porcentajes) según tipos de traumas en la infancia y número (porcentaje) de cada uno de los traumas en la infancia

\begin{tabular}{lll}
\hline Evento traumático en la infancia & $\begin{array}{l}\text { Número de sujetos con tipo de } \\
\text { traumas }(\%)\end{array}$ & Número de tipo de traumas \\
\hline Abuso físico & $17(49 \%)$ & $29(34.5 \%)$ \\
Abuso sexual & $15(46.8 \%)$ & $21(25 \%)$ \\
Muerte inesperada de familiar lamigo & $10(31.2 \%)$ & $12(14.2 \%)$ \\
Experiencia de estar cercano a ahogarse & $8(25 \%)$ & $9(10.7 \%)$ \\
Accidente de coche, tren o avión & $7(21 \%)$ & $7(8.3 \%)$ \\
\hline Total & $32^{*}$ & 84 \\
\hline${ }^{*}$ Nota: Muchos sujetos experimentaron distintos tipos de traumas por eso no suman $100 \%$
\end{tabular}


Tabla 3

Número promedio de experiencias traumáticas en la niñez y la adultez, con respecto a la presencia de alucinaciones y delirios

\begin{tabular}{lll}
\hline Variables & $\begin{array}{l}\text { Infancia } \\
\boldsymbol{M}(\boldsymbol{D} \boldsymbol{T})\end{array}$ & $\begin{array}{l}\text { Adultez } \\
\boldsymbol{M}(\boldsymbol{D} \boldsymbol{T})\end{array}$ \\
\hline Con alucinaciones $(\mathrm{n}=30)$ & $2.03(2.23)$ & $2.40(2.23)$ \\
Sin alucinaciones $(\mathrm{n}=41)$ & $0.56(0.95)$ & $1.95(1.59)$ \\
$U(p)$ & $370(.002)$ & $556(.484)$ \\
\hline Con delirios $(\mathrm{n}=45)$ & $1.58(2.02)$ & $2.40(2.18)$ \\
Sin delirios $(\mathrm{n}=26)$ & $0.50(.860)$ & $1.69(1.12)$ \\
$U(p)$ & $412.50(.024)$ & $491(.252)$ \\
\hline
\end{tabular}

Con relación al género, no hemos encontrados diferencias significativas entre número de hombres y mujeres con experiencias traumáticas en la infancia $\left(\chi_{(1)}^{2}=0.036, p=.850\right)$ y en la adultez $\left(\chi_{(1)}^{2}=0.893, p\right.$ $=.345)$, ni tampoco en el número de traumas padecidos por cada uno de ellos (infancia: $t_{(69)}=0.330, p=.742$; adultez: $\left.t_{(69)}=0.527, p=.600\right)$. Concretamente los hombres padecieron un media de 1.22 traumas en la infancia y 2.07 en la adultez y las mujeres padecieron 1.06 traumas en la infancia, y 2.35 traumas en la adultez.

Como se muestra en la Tabla 3, se dividió la muestra en pacientes con y sin alucinaciones por una parte, y pacientes con y sin delirios por otra. Los sujetos con alucinaciones habían experimentado un número significativamente mayor de experiencias traumáticas en la infancia $(U=370, p<.002)$ que los sujetos sin alucinaciones. Sin embargo, no habían experimentado significativamente más traumas en la edad adulta que los que no habían tenido ninguna experiencia alucinatoria $(U=556, p=.484)$. Por otra parte, hemos encontrado diferencias significativas entre los participantes con y sin delirios en el número de traumas en la infancia $(U=412.50, p=.024)$, pero no en la edad adulta $(U=491, p=.252)$.

En la Tabla 4, se observa que hay diferencias estadísticamente significativas entre los participantes con y sin alucinaciones en cuanto al tipo de traumas en la infancia, de manera que quienes sufrieron alucinaciones experimentaron más experiencias de abuso físico $(p=.004)$, amenazas serias a un familiar o amigo $(p=.003)$ y experiencias de estar próximos a ahogarse $(p=.046)$ que los sujetos sin alucinaciones. No obstante, aunque no se encontró diferencias significativas en relación con los abusos sexuales, sí había una tendencia a la significación $(p=.094)$. En cambio, ninguna de las situaciones traumáticas en la infancia estuvo estadísticamente relacionadas con la presencia o ausencia de delirios.

Tabla 4

Número promedio de tipos de experiencias traumáticas, con respecto a la presencia de alucinaciones y delirios

\begin{tabular}{|c|c|c|c|c|c|c|}
\hline Variables & $\begin{array}{l}\text { Abuso } \\
\text { físico } \\
M(D T)\end{array}$ & $\begin{array}{l}\text { Abuso } \\
\text { sexual } \\
M(D T)\end{array}$ & $\begin{array}{l}\text { Muerte } \\
\text { inesperada } \\
\text { de familiar / } \\
\text { amigo } \\
M(D T)\end{array}$ & $\begin{array}{l}\text { Experiencia } \\
\text { de estar } \\
\text { próximo a } \\
\text { ahogarse } \\
M(D T)\end{array}$ & $\begin{array}{l}\text { Accidente } \\
\text { de coche, } \\
\text { tren o avión } \\
M(D T)\end{array}$ & $\begin{array}{l}\text { Amenaza } \\
\text { seria a } \\
\text { familiar / } \\
\text { amigo } \\
M(D T)\end{array}$ \\
\hline $\begin{array}{l}\text { Con alucinaciones } \\
(n=30)\end{array}$ & $0.80(1.44)$ & $0.47(0.81)$ & $0.27(0.58)$ & $0.23(0.50)$ & $0.07(0.25)$ & $0.20(0.40)$ \\
\hline $\begin{array}{l}\text { Sin alucinaciones } \\
(n=41)\end{array}$ & $0.12(0.33)$ & $0.17(0.44)$ & $0.10(0.30)$ & $0.05(0.21)$ & $0.12(0.33)$ & $0.00(0.00)$ \\
\hline$U(p)$ & $429(.004)$ & $512.5(.094)$ & $548(.196)$ & $521(.046)$ & $581(.443)$ & $429(.003)$ \\
\hline $\begin{array}{l}\text { Con delirios } \\
(n=45)\end{array}$ & $0.56(1.23)$ & $0.38(0.74)$ & $0.22(0.51)$ & $0.18(0.44)$ & $0.11(0.31)$ & $0.13(0.34)$ \\
\hline $\begin{array}{l}\text { Sin delirios } \\
(n=26)\end{array}$ & $0.15(0.36)$ & $0.15(0.36)$ & $0.08(0.27)$ & $0.04(0.19)$ & $0.08(0.27)$ & $0.00(0.00)$ \\
\hline$U(p)$ & $494(.145)$ & $522(.291)$ & $524(.228)$ & $516(.133)$ & $507(.053)$ & $507(.644)$ \\
\hline
\end{tabular}


Respecto a las experiencias disociativas, también se realizaron los análisis comparando sujetos con y sin alucinaciones por una parte, y sujetos con y sin delirios por otra (véase la Tabla 5). La puntuación media de disociación fue significativamente mayor para los sujetos que tenían alucinaciones que los que no tenían $(p<.001)$, y también para los que tenían delirios en comparación con aquellos que no los tenían $(p=.003)$. Asimismo, los pacientes que habían experimentado traumas en la infancia o en la edad adulta se compararon con los que no habían experimentado dichas experiencias, encontrando únicamente diferencias significativas para pacientes con traumas en la infancia $(p=.009)$, esto es, los pacientes con traumas en la infancia presentaron significativamente niveles más altos en disociación que los pacientes sin traumas en este periodo de la vida.

Para finalizar, con respecto a la sexta hipótesis acerca de la predicción de las alucinaciones con respecto a las experiencias disociativas y traumáticas, los resultados apoyan la importancia de la despersonalización. Se tomaron como variables predictoras la presencia de traumas en la infancia y en la edad adulta (variables dicotómicas) en un primer paso; los factores de la escala de disociación (amnesia disociativa, absorción y despersonalización) como segundo paso, y la variable alucinaciones como criterio (dicotómica) (procedimiento introducir). La prueba de Hosmer y Lemeshow alcanza un valor Chi-cuadrado de 15.859 ( 8 g.1., $p=.044$ ). El resultado muestra una clasificación correcta del $85.9 \%$ de los casos (con alucinaciones y sin alucinaciones), explicando un $70.9 \%$ de la varianza ( $R^{2}$ de Nagelkerke). La variable que resulta significativa definitivamente es la despersonalización ( Wald $=12.008,1$ g.1., $p=.001$ ), $I C$ $95 \%$ 1.086-1.348, perdiendo la significación la absorción $($ Wald $=2.691,1$ g.1., $p=.101)$, más una constante significativa ( Wald $=4.846,1$ g.l., $p=.028$ ). La puntuación $\mathrm{B}$ positiva indica que la relación entre la despersonalización y las alucinaciones es directa. De las variables sobre los traumas, en el primer paso resultó significativa la relativa a traumas en la infancia $($ Wald $=$ $6.423,1$ g.l., $p=.011)$, si bien perdió la significación en el segundo paso $($ Wald $=2.666,1$ g.1., $p=.103)$.

Se realizó el mismo procedimiento con la variable delirios como criterio (dicotómico), siguiendo el mismo procedimiento de variables predictoras en dos pasos. De forma global, los resultados indican que ni los traumas ni las variables de disociación predicen los delirios. En el primer paso, resultó significativa la variable relativa a traumas en la infancia ( Wald $=4.124,1$ g.1., $p=.042)$, si bien perdió la significación en el segundo paso $($ Wald $=$ 2.051, 1 g.l., $p=.152$ ). La prueba de Hosmer y Lemeshow alcanza un valor Chi-cuadrado de 2.458 (8 g.l., $p=.964)$. El resultado muestra una clasificación correcta del $69 \%$ de los casos (con delirios y sin delirios), explicando un $23.1 \%$ de la varianza $\left(R^{2}\right.$ de Nagelkerke). Las variables relativas a la disociación no resultaron estadísticamente significativas, ni la despersonalización ( Wald $=2.206,1$ g.1., $p=.137$ ), ni absorción $($ Wald $=0.066,1$ g.1., $p=.798)$, ni amnesia disociativa $($ Wald $=1.633,1$ g.1., $p=.201)$.

\section{Discusión}

El objetivo de este trabajo fue estudiar la relación de dos tipos específicos de síntomas psicóticos positivos (las alucinaciones auditivas verbales y delirios) con experiencias traumáticas (en la infancia y la edad adulta) y disociativas.

Tabla 5

Puntuaciones medias (DT) en la Escala de Experiencias Disociativas DES-II para los participantes con trauma, alucinaciones y delirios

\begin{tabular}{lllll}
\hline Variables & SI & NO & $\boldsymbol{t}(\mathbf{g l})$ & $\boldsymbol{p}$ \\
\hline Alucinaciones & $\boldsymbol{M}(\boldsymbol{D} \boldsymbol{T})$ & $\boldsymbol{M}(\boldsymbol{D} \boldsymbol{T})$ & $6.24(69)$ & .001 \\
Delirios & $28.05(12.64)$ & $11.85(9.24)$ & $3.07(66.53)$ & .003 \\
Trauma en la infancia & $21.93(14.21)$ & $13.10(9.87)$ & $2.72(49.96)$ & .009 \\
Trauma en la adultez & $23.46(15.67)$ & $14.79(9.82)$ & $1.01(69)$ & .312 \\
\hline
\end{tabular}


Hemos encontrado que casi la mitad de los sujetos (45.1\%) de nuestro estudio habían padecido algún tipo de experiencia traumática en la infancia, y casi la totalidad de la muestra (80.3\%) habían padecido traumas en la edad adulta. Estos datos vienen a mostrar por un lado, que las personas con psicosis, en general, son víctimas de experiencias traumáticas (e.g., Longden, Madill y Waterman, 2011), y por otro, apoya la hipótesis de aquellos autores que defienden que las personas que padecen traumas en la infancia (como abusos físicos y sexuales) tienen una probabilidad mayor de sufrir traumas de adultos (Cloitre, Tardiff, Marzuk y Leon, 1996; Muenzenmaier et al., 1993). Pero también hay que destacar que algunos de nuestros sujetos que no padecieron traumas en la infancia, sí tuvieron experiencias traumáticas en la adultez. Esto también confirma la idea de la vulnerabilidad de las personas adultas con psicosis de ser blanco del maltrato de otros, y a su vez, de su sensibilidad extrema a estas situaciones y al estigma social (Garety, Kuipers, Fowler, Freeman y Bebbington, 2001). Asimismo, vemos también que el tipo de experiencias traumáticas en la infancia que padecen con mayor frecuencia son de tipo interpersonal, esto es, abusos físicos y sexuales. Este dato está corroborado en numerosas investigaciones, y algunos autores plantean que pueden ser factores causales de la psicosis (Varese et al., 2012).

Respecto a la primera hipótesis, hemos encontrado que los sujetos con alucinaciones auditivas han padecido más acontecimientos traumáticos en la infancia. Estos datos son consistentes con los resultados obtenidos en otros estudios (e.g., Perona-Garcelán et al, 2010; Read et al., 2003; Ross, Anderson y Clark, 1994). Sin bien parece que existe una relación entre traumas en la infancia y alucinaciones, no está clara la relación entre traumas en la infancia y delirios (Perona-Garcelán et al. 2010; Read, Goodman, Morrison, Ross y Aderhold, 2004). No obstante, en el estudio actual hemos encontrado que los sujetos con delirios presentaron significativamente un mayor número de traumas en la infancia que los sujetos sin delirios. En una revisión de la literatura sobre el tema, Read et al. (2003) propusieron la idea de que los traumas en la infancia estarían más fuertemente relacionados con las alucinaciones que con los delirios. Nuestros resultados son compatibles con esta idea, pues el tamaño del efecto obtenido entre alucinaciones y traumas en la infancia es mayor que entre delirios y traumas en la infancia ( $d$ de Cohen 1.41 y 0.70 respectivamente).

En nuestra segunda hipótesis, proponíamos que no había diferencias significativas entre sujetos con y $\sin$ alucinaciones, y delirios, con relación a los traumas padecidos en la edad adulta. Los resultados confirman esta hipótesis, siendo coherente con los obtenidos por Perona-Garcelán et al. (2010). Probablemente, mientras en la infancia el trauma (y las experiencias adversas en general) sea un factor de riesgo que predispone a estas personas a padecer sintomatología psicótica positiva (Varese et al., 2012), la presencia de traumas en la edad adulta pudiera estar más relacionada con personas con un trastorno mental severo en general, y tal vez tuviera que ver con el estado de vulnerabilidad social en el que se encuentran después de años de padecer el trastorno. De todas formas, podríamos especular, junto con Read, van Os, Morrison y Ross (2005), que los traumas en la edad adulta podrían ser un mediador potencial entre los traumas en la infancia y la psicosis. Hipótesis que sería interesante confirmar en futuros estudios.

Respecto al tipo específico de traumas, nuestra hipótesis se ha cumplido parcialmente. Por un lado, hemos encontrado que los sujetos con alucinaciones auditivas padecieron más traumas de tipo interpersonal (como el abuso físico y la amenaza a un familiar o a un amigo) que los sujetos sin este tipo de síntomas; pero por otro lado, no hemos encontrado diferencias significativas en cuanto a esta variable entre sujetos con $\mathrm{y}$ sin delirios. Es probable, tal y como encontraron Bentall, Wickham, Shevlin y Varese (2012), que diferentes síntomas psicóticos estén asociados a adversidades también diferentes en la infancia. Esto podría explicar por qué no hemos encontrado diferencias entre sujetos con y sin delirios respecto a traumas específicos. En el estudio mencionado de Bentall et al. (2012), el abuso sexual estaba asociado específicamente con las alucinaciones, y el maltrato institucional en la infancia con la paranoia. Es probable 
que otros tipos de traumas no descritos en la escala utilizada en nuestro estudio, estén relacionados con las ideas delirantes. No obstante, no debemos descartar que otra forma de explicar esta carencia de resultado sea debido al tamaño de la muestra.

En cuanto a la cuarta hipótesis, hemos encontrado que tanto las alucinaciones auditivas como las ideas delirantes están relacionadas con la presencia de síntomas disociativos. Estos resultados son coherentes con los obtenidos en otros estudios en los que encontraron, por un lado, una correlación positiva entre alucinaciones y delirios con disociación (PeronaGarcelán et al., 2010), y por otro, entre alucinaciones con absorción y despersonalización (Perona-Garcelán et al. 2008, 2012). Estos resultados apoyan por un lado la hipótesis de que las alucinaciones auditivas pueden tener una base disociativa (Moskowitz y Corsten, 2007; Perona-Garcelán, Pérez-Álvarez, García-Montes y Cangas, 2015) y por otro, el papel que pueden tener la disociación como fuente de experiencias anómalas que favorece que la persona con psicosis las interprete de forma delirante (Garety et al., 2001).

Nuestros resultados también confirman la hipótesis quinta planteada en el presente estudio. Los sujetos que han padecido traumas en la infancia, muestran niveles de disociación significativamente más severos que los sujetos que no han sufrido este tipo de experiencias traumáticas. Sin embargo, hemos encontrado que los traumas en la adultez no están significativamente asociados con la disociación. Estos resultados, junto con lo descrito anteriormente respecto a la relación entre alucinaciones y disociación, permiten plantearnos la función mediadora que puede tener la disociación entre los traumas en la infancia y los síntomas psicóticos positivos. Hasta la fecha se han realizado dos estudios que confirman parcialmente esta idea. En este sentido, Varese, Barkus y Bentall (2012) encontraron que la disociación mediaba entre los abusos sexuales en la infancia y la propensión a las alucinaciones en pacientes psicóticos, y Perona-Garcelán, CarrascosoLópez et al (2012) encontraron que si bien las disociación, y más específicamente la despersonalización, mediaba entre los traumas en la infancia y la severidad de las alucinaciones, no mediaba entre traumas y severidad de los delirios. Esto viene a reforzar la hipótesis planteada de la importancia que tiene la disociación en la comprensión de las alucinaciones auditivas como experiencias de base disociativa.

Por último, y en relación con lo anteriormente expuesto, hemos encontrado que de las variables utilizadas en este estudio, el factor de despersonalización de la DES-II, frente a los otros dos factores (amnesia disociativa y absorción) y los traumas, es el único factor que predice la severidad de las alucinaciones y no de los delirios. Estos resultados son coincidentes con los de Kilcommons y Morrison (2005), y Perona-Garcelán y colaboradores (Perona-Garcelán et al., 2008; Perona-Garcelán, García-Montes, et al., 2012) en sujetos con psicosis y en Perona-Garcelán et al. (2013) en sujetos sanos con propensión a las alucinaciones (junto con la absorción), lo cual viene a confirmar la importancia de las experiencias disociativas para la comprensión de las alucinaciones auditivas.

Las conclusiones de este trabajo, debido a las características metodológicas del mismo, deben ser matizadas y tomadas con precaución. Este estudio puede ser considerado como un estudio preliminar que debería replicarse con una muestra mayor de personas, y en la medida de lo posible, con grupos de control con sujetos sin patología psiquiátrica con alta y baja predisposición a las alucinaciones y delirios. El que nuestra muestra esté formada por un mayor número de hombres que de mujeres, es también una limitación que podría explicar el relativamente bajo número de traumas reportados por nuestros sujetos en comparación con otros estudios (Read, Fink, Rudegeair, Felitti, y Whitfield, 2008). Por otro lado, sería deseable usar otras medidas disociativas que no estén contaminadas por ítems que podrían también medir sintomatología psicótica (por ejemplo, el ítem 27 de la DES-II evalúa alucinaciones). La escala utilizada para medir el trauma está basada en el recuerdo del propio paciente. Ello puede sesgar los resultados obtenidos, por lo que sería deseable contrastar tal 
información con la de familiares y cuidadores. De todas formas, hay estudios que muestran que los informes sobre historias de trauma en enfermos mentales graves son generalmente fiables (Fisher et al., 2011; Goodman, et al., 1999).

En relación con las aplicaciones clínicas de nuestro estudio, los presentes hallazgos apoyan las recomendaciones hecha por otros investigadores que aconsejan preguntar a los pacientes con psicosis sobre las experiencias traumáticas padecidas en la infancia y en la adultez, de forma que faciliten una mejor comprensión de la fenomenología de sus alucinaciones y delirios, y una mejor formulación de los objetivos de tratamiento (Read, Hammersley y Rudegeair, 2007).

Para terminar, hay que destacar que el presente estudio, a pesar de las limitaciones antes expuestas, entra a formar parte del cada vez mayor grupos de trabajos que destacan la relación tan relevante que existe entre trauma y disociación en psicosis. Apoyando los hallazgos de otros autores de que los traumas en la infancia pueden estar relacionados con la etiología de algunos tipos de psicosis. De hecho, tendría sentido prever que estas investigaciones conduzcan a medio y largo plazo a modificar nuestra concepción de los que hoy entendemos por esquizofrenia y los criterios que actualmente utilizamos para su diagnóstico. Autores como Ross (2004), defienden un tipo disociativo de esquizofrenia caracterizado por la presencia de pocos síntomas negativos y ausencia de trastornos del pensamiento, más síntomas positivos y mayor comorbilidad en los ejes 1 y 2 (incluyendo trastornos disociativos y trastornos por estrés postraumáticos), más experiencias de trauma, mayores puntuaciones en escalas de disociación, mayor relación con las voces y mayor poder ejecutivos de éstas, etc. (Ross, 2004).

\section{Referencias}

Aleman, A. y LarØi, F. (2008). Hallucinations: The science of idiosyncratic perception. Washington: American Psychological Association.

American Psychiatric Association. (1994). Diagnostic and statistical manual for mental disorders (4a Ed.). Washington, DC: Autor.

Bendall, S., Jackson, H. J., Hulbert, C. A. y McGorry, P. D. (2008). Childhood trauma and psychotic disorders: a systematic, critical review of the evidence. Schizophrenia Bulletin, 34(3), 568-579. doi: http://dx.doi.org/10.1093/schbul/sbm121

Bentall, R. P. (1990). The illusion of reality: a review and integration of psychological research on hallucinations. Psychological Bulletin, 107(1), 82-95.

Bentall, R. P., Wickham, S., Shevlin, M. y Varese, F. (2012). Do Specific Early-Life Adversities Lead to Specific Symptoms of Psychosis? A Study from the 2007. The Adult Psychiatric Morbidity Survey. Schizophrenia Bulletin, 38(4), 734-740. doi: http://dx.doi.org/10.1093/schbul/sbs049

Bobes, J., Calcedo-Barba, A., García, M., François, M., Rico-Villademoros, F., González, M. P., ... Bousoño, M. (2000). Evaluation of the psychometric properties of the Spanish version of five questionnaires for the assessment of post-traumatic stress. Acta Española de Psiquiatría, 28, 207-218.

Carlson, E.B. y Putnam, F. W. (1993). An update on the Dissociative Experiences Scale. Dissociation, 6, 1627.

Cascardi, M., Mueser, K., Degiralomo, J. y Murrin, M. (1996). Physical aggression against psychiatric inpatients by family members and partners. Psychiatry Services, 47, 531-533.

Cloitre, M., Tardiff, K., Marzuk, P. M. y Leon, A. C. (1996). Childhood abuse and subsequent sexual assault among female inpatients. Journal of Traumatic 
Stress, $\quad 9, \quad 473-482$. http://dx.doi.org/10.1002/jts.2490090306

Davidson, J. R. E., Hughes, D. y Blazer, D. G. (1990). Traumatic experiences in psychiatric patients. Journal of Trauma Stress, 3, 459-475.

Fisher, H. L., Craig, T. K., Fearon, P., Morgan, K., Dazzan, P., Lappin, J., ... Morgan, C. (2011). Reliability and comparability of psychosis patients' retrospective reports of childhood abuse. Schizophrenia Bulletin, 37(3), 546-553. doi: http://dx.doi.org/10.1093/schbul/sbp103

Frith, C. D. (1995). La esquizofrenia. Un enfoque neuropsicológico. Barcelona, España: Ariel (edición original, 1992).

Garety, P. A., Kuipers, E., Fowler, D., Freeman, D. y Bebbington, P. E. (2001). A cognitive model of positive symptoms of psychosis. Psychological Medicine, $\quad 31$, 189-195. http://dx.doi.org/10.1017/S0033291701003312

Goodman, L. A., Thompson, K. M., Weinfurt, K., Corls, S., Ackert, P., Mueser, K.T., ...Rosenberg, S. D. (1999). Reliability of reports of violent victimization and posttraumatic stress disorder among men and women with serious mental illnes. Journal of Trauma and Stress, 12(4), 587-599.

Icaran, E., Colom, R. y Orengo-García, F. (1996). Estudio de validación de la escala de experiencias disociativas con muestra de población española. Actas Luso-Española de Neurología, Psiquiatría y Ciencias Afines, 24(1), 7-10.

Kay, S. R., Opler, L. A. y Lindenmayer, J. P. (1988). Reliability and validity of the Positive and Negative Syndrome Scale for schizophrenics. Psychiatry Research, 23, 99-110.

Kilcommons, A. M. y Morrison, A. P. (2005). Relationships between trauma and psychosis: An exploration of cognitive and dissociative factors. Acta
Psychiatrica Scandinavica, 112, 351-359. doi: http://dx.doi.org/10.1111/j.1600-0447.2005.00623.x

Longden, E., Madill, A. y Waterman, M. G. (2012). Dissociation, trauma, and the role of lived experience: Toward a new conceptualization of voice hearing. Psychological Bulletin, 138(1), 28-76. doi: http://dx.doi.org/10.1037/a0025995

Moskowitz, A. y Corstens, D. (2007). Auditory hallucinations: Psychotic symptom or dissociative experience. Journal of Psychological Trauma, 6(2-3), 35-63. doi: http://dx.doi.org/10.1300/J513v06n02_04

Muenzenmaier, K., Meyer, I., Struening, E. y Ferber, J. (1993). Childhood abuse and neglect among women outpatients with chronic mental illness. Hospital and Community Psychiatry, 44, 666-670.

Offen, L., Waller, G. y Thomas, G. (2003). Is reported childhood sexual abuse associated with the psychopathological characteristics of patients who experience auditory hallucinations? Child Abuse and Neglect, 27, 919-927. doi: http://dx.doi.org/10.1016/S0145-2134(03)00139-X

Peralta, V. y Cuesta, M. J. (1994). Validación de la Escala de los Síndromes Positivo y Negativo (PANSS) en una muestra de esquizofrénicos españoles. Actas Luso-Española de Neurología, Psiquiatría y Ciencias Afines, 22(4), 171-177.

Perona-Garcelán, S., Pérez-Álvarez, S., García-Montes, J. M. y Cangas, A, (2015). Auditory Verbal Hallucinations as Dialogic Experiences. Journal of Constructivist Psychology, 28(3), 264-280. doi: http://dx.doi.org/10.1080/10720537.2014.938847

Perona-Garcelán, S., Cuevas-Yust, C., García-Montes, J. M., Pérez-Álvarez, M., Ductor-Recuerda, M. J., SalasAzcona, R.,... Rodríguez-Martín, B. (2008). Relationship between self-focused attention and dissociation in patients with and without auditory hallucinations. Journal of Nervous and Mental Disease, 196(3), 190-197. doi: http://dx.doi.org/10.1097/NMD.0b013e318165c7c1. 
Perona-Garcelán, S., García-Montes, J. M., CuevasYust, C., Pérez-Álvarez, M., Ductor-Recuerda, M. J.,... Gómez-Gómez, M. T. (2010). A preliminary exploration of trauma, dissociation, and positive psychotic symptoms in a Spanish sample. Journal of Trauma \& Dissociation,11(3), 284-292. doi: http://dx.doi.org/10.1080/15299731003786462.

Perona-Garcelán, S., García-Montes, J. M., DuctorRecuerda, M. J., Vallina-Fernández, O., CuevasYust, C., Pérez-Álvarez, M., ...Gómez-Gómez, M.T. (2012). Relationship of metacognition, absorption, and depersonalization in patients with auditory hallucinations. British Journal of Clinical Psychology, 51(1), 100-118. doi: http://dx.doi.org/10.1111/j.2044-8260.2011.02015.x

Perona-Garcelán, S., Carrascoso- López, F., GarcíaMontes, J. M., Ductor-Recuerda, M. J., LópezJiménez, A. M., ....Gómez-Gómez, M. T. (2012). Dissociative Experiences as Mediators Between Childhood Trauma and Auditory Hallucinations. Journal of Traumatic Stress, 25(3), 323-329. doi: http://dx.doi.org/10.1002/jts.21693

Perona-Garcelán, S., García-Montes, J. M., RodríguezTestal, J. F., Ruiz-Veguilla, M., Benítez-Hernández, M. M., López-Jiménez, A.M., ... Pérez-Álvarez, M. (2013). Relationship of absorption, depersonalization and self-focused attention in subjects with and without hallucination proneness. Cognitive Neuropsychiatry, 18(5), 422-436. doi: http://dx.doi.org/10.1080/13546805.2012.728133

Read, J. (1997). Child abuse and psychosis: a literature review and implications for professional practice. Professional Psychology: Research and Practice 28, 448-456. 7028.28 .5 .448

Read, J., Agar, K., Argyle, N. y Aderhold, V. (2003). Sexual and physical abuse during childhood and adulthoods as predictors of hallucinations, delusions and thought disorder. Psychology and Psychotherapy: Theory, Research and Practice, 76, $1-22$.
Read, J., Goodman, L., Morrison, A., Ross, C. A. y Aderhold, V. (2004). Childhood trauma, loss and stress. En, J. Read, L. R. Mosher y R. P. Bentall (Eds.), Models of madness: psychological, social and biological approaches to schizophrenia (pp. 223-252). Hove, UK: Brunner-Routledge.

Read, J., van Os, J., Morrison, A. P. y Ross, C. A. (2005). Childhood trauma, psychosis and schizophrenia: a literature review with theoretical and clinical implications. Acta Psychiatrica Scandinavica, 112, 330-350. doi: http://dx.doi.org/10.1111/j.16000447.2005.00634.x

Read, J., Hammersley, P. y Rudegeair, T. (2007). Why, when and how to ask about child abuse. Advances in Psychiatric Treatment, 13, 101-110. doi: http://dx.doi.org/10.1192/apt.bp.106.002840

Read, J., Fink, P. J., Rudegeair, T., Felitti, V. y Whitfield, C. L. (2008). Child maltreatment and psychosis: A return to a genuinely integrated bio-psycho-social model. Clinical Schizophrenia \& Related Psychoses, 2(3), 235-254.

Ritsher, J., Coursey, R. y Farrell, E. (1997). A survey on issues in the lives of women with severe mental illness. Psychiatry Services, 48, 1273-1282.

Ross, C. A. (2004). Schizophrenia. Innovations in diagnosis and treatment. New York: HMTP.

Ross, C. A. y Keyes, B. (2004). Dissociation and schizophrenia. Journal of Trauma and Dissociation, $5,69-83$.

Ross, C. A., Anderson, G. y Clark, P. (1994). Childhood abuse and positive symptoms of schizophrenia. Hospital and Community Psychiatry, 45, 489-491.

Sar, V., Taycan, O., Bolat, N., Özmen, M., Duran, A. Öztörk, E. y Ertem-Vehid, H. (2010). Childhood trauma and dissociation in schizophrenia. Psychopathology, 43, 33-40. doi: http://dx.doi.org/10.1159/000255961. 
Schäfer, I., Harfst, T., Aderhold, V., Briken, P., Lehmann, M., Moritz, ... Naber, D. (2006). Childhood trauma and dissociation in female patients with schizophrenia spectrum disorders. An exploratory study. Journal of Nervous and Mental Disease, 194(2), 135-138.

Schenkel, L. S., Spaulding, W. D., DiLillo, D. y Silverstein, S. M (2005). Histories of childhood maltreatment in schizophrenia: Relationships with premorbid functioning, symptomatology, and cognitive déficits. Schizophrenia Research 76, 273286.

Varese, F, Barkus, E. y Bentall R. P. (2012). Dissociation mediates the relationship between childhood trauma and hallucination proneness. Psychological Medicine, 42(5), 1025-1036. doi: http://dx.doi.org/10.1017/S0033291711001826

Varese, F., Smeets, F., Drukker, M., Lieverse, R., Lataster, T., Viechtbauer, W., ... Bentall, R. P. (2012). Childhood Adversities Increase the Risk of Psychosis: A Meta-analysis of Patient-Control, Prospective- and Cross-sectional Cohort Studies. Schizophrenia Bulletin, 38(4), 661-671. doi: http://dx.doi.org/10.1093/schbul/sbs050 
